\title{
Utility of Flexible Bronchoscopy for Airway Foreign Bodies Removal in Adults
}

\author{
Jose N. Sancho-Chust *, Virginia Molina, Sandra Vañes, Ana M. Pulido, Lia Maestre and \\ Eusebi Chiner ${ }^{\mathbb{D}}$
}

Secció de Pneumologia, Hospital Universitari Sant Joan d'Alacant, Ctra Alacant-València s/n, 03550 Sant Joan d'Alacant, Spain; virginia_molpe@hotmail.com (V.M.); sandravanesbanos@gmail.com (S.V.); pulidawork@gmail.com (A.M.P.); lia_maestre@yahoo.es (L.M.); echinervives@gmail.com (E.C.)

* Correspondence: norbertosancho@gmail.com; Tel.: +34-965658750

Received: 11 April 2020; Accepted: 7 May 2020; Published: 10 May 2020

\begin{abstract}
Foreign body aspiration is relatively infrequent in adults. Airway foreign bodies (AFBs) can be removed by flexible bronchoscopy (FB) or rigid bronchoscopy (RB). We performed a retrospective analysis of FBs performed in our centre over a 25 year period, focusing on the procedures that revealed an AFB during the examination stage. We recorded demographic data, clinical characteristics and radiological and bronchoscopic findings. During the study period, 12,588 FBs were performed in adults. Airway foreign bodies were identified in 32 of these cases, giving a prevalence of $0.25 \%$. The most frequent clinical presentation was cough, sputum and fever. The most frequent radiological findings were alveolar infiltrates and atelectasis. In $94 \%$ of cases, AFBs were removed successfully by FB; RB was necessary in only $6 \%$ of cases. There were no FB-related complications. The most common AFB location was the right bronchial tree (69\%). We classified AFBs as organic (85\%: animal $57 \%$; vegetable $28 \%$ ), inorganic (6\%) and iatrogenic (9\%). Bronchial infection occurred in $51 \%$ of cases, and Actinomyces spp. was the most common causal microorganism. In conclusion, AFBs are a rare entity with nonspecific clinical presentation, most AFBs were organic, and FB is a safe and effective method for AFB removal.
\end{abstract}

Keywords: bronchoscopy; flexible bronchoscopy; airway foreign bodies

\section{Introduction}

Accidental foreign body aspiration is less common in adults than in children, and there are major differences in clinical presentation between these two populations. Most publications on the topic are case reports; there are very few case series. Determining the exact prevalence of airway foreign bodies (AFBs) is therefore problematic, but they are thought to account for between $0.2 \%$ and $0.33 \%$ of all bronchoscopies [1-5].

There are documented cases of AFB managed, without much success, by cough manoeuvres, changes in position and ingestion of olive oil or emetics [6].

Endoscopic AFB removal was first described by Gustav Killian, the "father of bronchoscopy". In 1898, he reported how he had used the first tracheoscope and a pair of forceps to extract a pig bone from a German farmer's airway [7].

This innovation completely changed the medical management of AFBs, and rigid bronchoscopy (RB) was used successfully for almost a century [8]. After the invention of flexible bronchoscopy (FB) by Shigeto Ikeda, in 1974 Donald Zavala and Mitchell Rhodes described the first successful application of this technique for AFB removal in artificial models and animals (dogs) [9].

The use of FB quickly extended for this indication with case series recording its success $[3,10]$. In recent years, researchers have explored the use of FB as the method of choice for AFB removal in 
adults [11]. Studies have demonstrated the efficacy and safety of this technique in inorganic AFB removal which normally involves a greater risk of airway complications [12,13].

The objective of our study was to measure the prevalence of AFBs in adults undergoing bronchoscopy, to describe the clinical and endoscopic characteristics of AFBs and to determine the efficacy and safety of FB for AFB removal.

\section{Experimental Section}

\subsection{Study Type}

This is a retrospective analysis of the database of all bronchoscopies performed in the Pulmonology Department in the period from January 1994 to October 2019 in patients aged over 18 years. The university hospital where the procedures were performed serves a population of some 250,000 inhabitants with slight variations during the study period. The bronchoscopists in our hospital perform bronchoscopies in the morning, and none work out of hours.

We collected data on the FBs that revealed an AFB during the examination stage and then reviewed the patients' medical records.

\subsection{Material Used}

The material used in the procedures evolved during the rather long study period, owing to technological improvements in the field of respiratory endoscopy.

Five different experienced bronchoscopists performed the examinations, along with the pulmonology residents who intervened as part of their training programme.

The bronchoscopists used different models of flexible bronchoscopes from the brands Pentax@ (e.g., EB-1570k and EB15-J10) and Fujifilm @ (e.g., EB530H, EB530S and EB530T). Most bronchoscopes had a distal outer diameter of between $49 \mathrm{~mm}$ and $54 \mathrm{~mm}$, with a $2.0 \mathrm{~mm}$ working channel. The therapeutic bronchoscopes were slightly bigger with a distal outer diameter of around $58 \mathrm{~mm}$ and a $2.8 \mathrm{~mm}$ working channel.

The instruments used in the FBs to remove the AFBs also varied and included standard bronchial biopsy forceps (both with and without teeth), foreign body forceps (alligator forceps) and foreign body retrieval baskets.

The samples obtained by bronchial suction were sent to the Microbiology Department for the usual staining and cultures. Quantitative cultures showing more than $1.10^{5}$ UFC were considered to be significant.

After examining the airway and locating the foreign body, the bronchoscopist removed it with the usual technique, securing it with the forceps or basket and removing it by withdrawing the bronchoscope and instrument together. If the AFB had sharp parts, these were directed towards the forceps or basket as far as possible, to minimise damage to the airway walls.

The FBs were performed in the Bronchoscopy Room of the Pulmonology Department with the assistance of the nursing and auxiliary staff. Some patients were put under minimal or conscious sedation with midazolam, usually at a dose of 1 to $5 \mathrm{mg}$. The local anaesthetic lidocaine was applied topically to the nostrils and with the bronchoscope to the rest of the airway using the "spray-as-you-go" technique [14].

In cases requiring RB, the bronchoscopist used a Wolf $\subseteq$ rigid bronchoscope with the standard accessories, including RB forceps which are considerably larger that FB forceps.

The RBs were performed in the operating theatre with the assistance of the usual anaesthesia team, who put the patient under propofol-induced deep sedation.

\subsection{Data Collection}

After identifying the cases with AFB diagnosis, we collected the following data regarding the endoscopic examination: indication of the examination, type of bronchoscope (FB or RB), point of entry, 
location of AFB, type of instrument used for removal, presence of histological analysis, microbiological results of the samples obtained, need for RB or surgery, and endoscopy-related complications (bleeding, respiratory failure, pneumothorax, cardiac arrest). We identified the foreign body types, classifying them as organic (of animal or vegetable origin), inorganic (minerals, metals, etc.) or iatrogenic (resulting from a medical intervention, e.g., dental prostheses).

Finally, we reviewed the medical records of each patient, collecting data related to age; sex; psychiatric comorbidities (personality disorder, schizophrenia, bipolar disorder); whether aspiration was voluntary; presence of abnormal radiological findings and their location; type and duration of symptoms; and complications due to the AFB, both short-term (pulmonary infections) and long-term (pulmonary sequelae after AFB removal).

\subsection{Statistical Analysis}

We expressed the numerical variables as means with standard deviations and the categorical variables as percentages. For all calculations we used the statistical package IBM SPSS (version 15.0; SPSS; Chicago, IL, USA).

\section{Results}

During the study period of 25 years and 10 months (310 months), 12,588 FBs were performed in adults. Airway foreign bodies were identified in 32 of these cases, giving a prevalence of $0.25 \%$.

Table 1 shows the demographic, clinical and radiological characteristics of the patients with AFB. In most cases, aspiration of a foreign body was an accidental finding. Suspected AFB was the indication for bronchoscopy in only $28 \%$ of these cases; the most common indication was pneumonia. There was no case of suspected AFB that could not be verified through FB examination. The most common clinical presentation was the triad of cough, sputum and fever with a mean duration of symptoms of 14 days before presentation. There were abnormal radiological findings in $94 \%$ of cases, the most common being alveolar infiltrates and atelectasis. The follow-up examinations showed no late complications of FB.

Table 1. Clinical and radiological characteristics of patients with airway foreign bodies (AFBs).

\begin{tabular}{lc}
\hline Parameter & Value \\
\hline Age & $63 \pm 12(32-81)$ \\
\hline Men & $53 \%$ \\
\hline Psychiatric comorbidities & $25 \%$ \\
\hline Voluntary foreign body aspiration & $6 \%$ \\
\hline Bronchoscopy indication: & \\
- Pneumonia & $35 \%$ \\
- Suspected AFB & $28 \%$ \\
- Atelectasis & $28 \%$ \\
- Suspected lung cancer & $3 \%$ \\
- Haemoptysis & $3 \%$ \\
- COPD & $3 \%$ \\
\hline Clinical presentation: & \\
- Cough, sputum and fever & $34 \%$ \\
- Dyspnoea & $25 \%$ \\
- Cough & $22 \%$ \\
- No symptoms & $16 \%$ \\
- Haemoptysis & $3 \%$ \\
\hline
\end{tabular}


Table 1. Cont.

\begin{tabular}{lc}
\hline Parameter & Value \\
\hline Duration of symptoms before presentation & $14 \pm 17(0-60)$ \\
\hline Radiological findings: & \\
- Alveolar infiltrate & $38 \%$ \\
- Atelectasis & $38 \%$ \\
- Calcific density & $18 \%$ \\
- No findings & $6 \%$ \\
\hline Sequelae after removal & $0 \%$ \\
\hline
\end{tabular}

Data are presented as number of patients (\%) or mean \pm SD (range). COPD: Chronic Obstructive Pulmonry Disease.

Table 2 shows the general characteristics of the FBs. In most cases, the bronchoscopist used an instrument designed specifically for foreign bodies (i.e., forceps or basket) to remove the AFB, although standard biopsy forceps were used in $31 \%$ of cases. The rate of success of FB for AFB removal was $94 \%$. Flexible bronchoscopy failed in the remaining $6 \%$ of cases because the AFB was too large to pass through the glottis. It remained in the bronchial tree and was successfully removed by RB in a later procedure. No cases required surgery, and there were no FB-related complications.

Table 2. Characteristics of flexible bronchoscopy (FB) in patients with an AFB.

\begin{tabular}{lc}
\hline Parameter & Occurrence \\
\hline Point of entry: & \\
- Mouth & $58 \%$ \\
- Nose & $39 \%$ \\
- Tracheostomy & $3 \%$ \\
\hline Instrument used: & \\
- Foreign body forceps & $53 \%$ \\
- Standard biopsy forceps & $31 \%$ \\
- Foreign body basket & $16 \%$ \\
\hline Histological confirmation & $13 \%$ \\
\hline Result of FB: & \\
- Removed & $94 \%$ \\
- Failure and need for RB & $6 \%$ \\
- Failure and referral to Thoracic Surgery & $0 \%$ \\
\hline Complications of the procedure & $0 \%$ \\
\hline \multicolumn{2}{c}{ Data are presented as number of patients $(\%)}$.
\end{tabular}

Table 3 shows the location of the AFBs in the airway. The most common location was the right bronchial tree (69\% of cases), followed by the left bronchial tree (25\%). Only $6 \%$ of AFBs were in the trachea or larynx.

Table 4 shows the types of AFBs removed. Organic AFBs accounted for $85 \%$ of cases, and of this group, $57 \%$ were of animal origin (mainly bones) and $28 \%$ of vegetable origin (mainly seeds). Inorganic AFBs accounted for $6 \%$ of cases (a ring and a metal cross). The remaining 9\% were iatrogenic AFBs, namely, two dental prosthesis and a dental implant. Figure 1 shows some images of AFBs removed by FB. 
Table 3. Location of the foreign bodies in the airway.

\begin{tabular}{lc}
\hline Location & Occurrence \\
\hline Larynx & $3 \%$ \\
Trachea & $3 \%$ \\
Right main bronchus & $13 \%$ \\
Right upper lobe bronchus & $3 \%$ \\
Bronchus intermedius & $19 \%$ \\
Middle lobe bronchus & $6 \%$ \\
Right lower lobe bronchus & $28 \%$ \\
Left main bronchus & $3 \%$ \\
Left upper lobe bronchus & $9 \%$ \\
Left lower lobe bronchus & $11 \%$ \\
\hline
\end{tabular}

Data are presented as number of patients (\%).

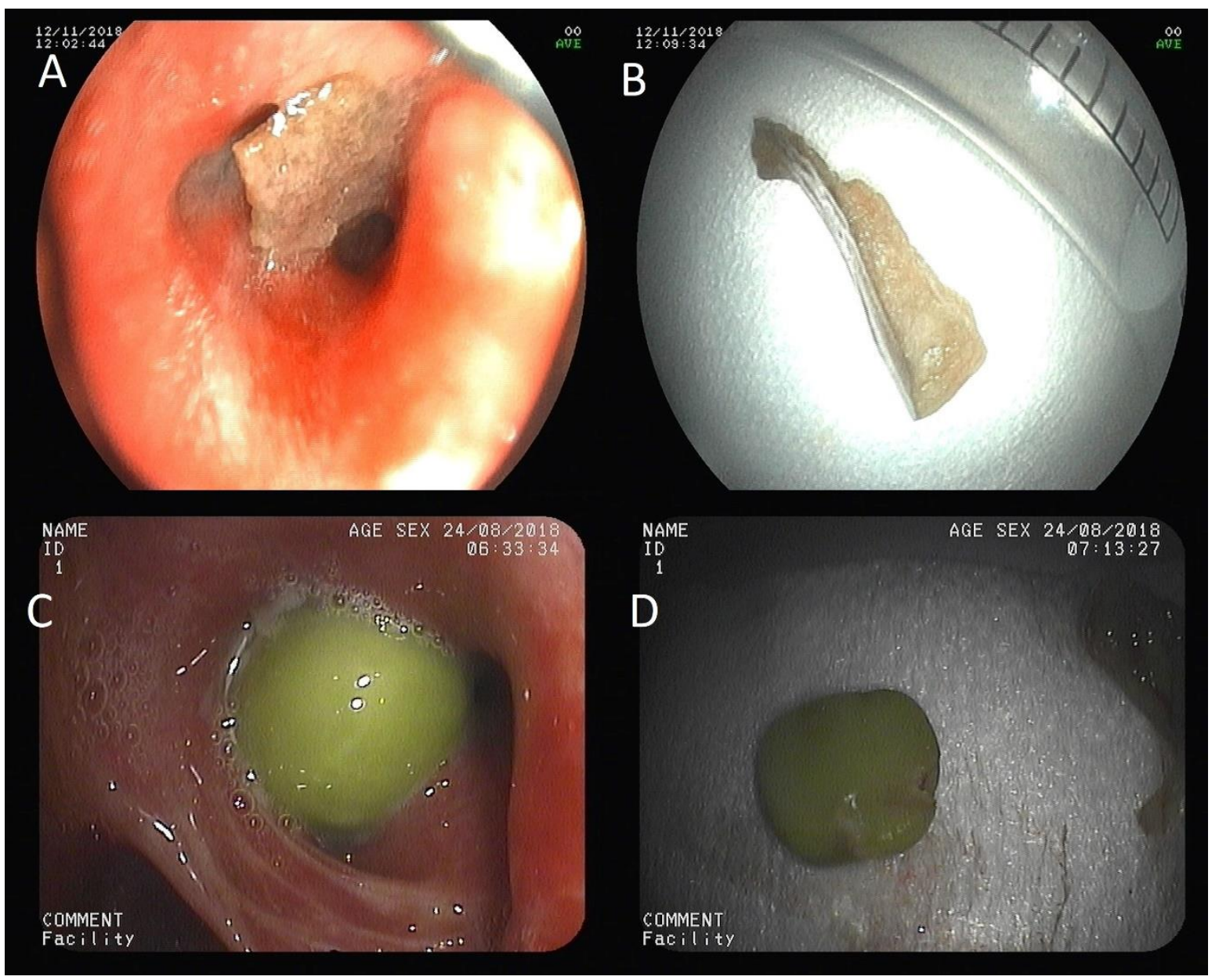

Figure 1. Examples of organic AFBs removed by FB. (A) pork rind located in the right lower lobe bronchus; (B) pork rind after removal by FB; (C) pea located in the left upper lobe bronchus; (D) pea after removal. FB: flexible bronchoscopy. AFB: airway foreign body. 
Table 4. Classification and specifications of AFB types.

\begin{tabular}{lc}
\hline AFB Type & Occurrence \\
\hline ANIMAL & \\
\hline Animal bone & $48 \%$ \\
Pork lard & $3 \%$ \\
Snail & $3 \%$ \\
Nail & $3 \%$ \\
\hline VEGETABLE & \\
\hline Seed & \\
- Pea & $10 \%$ \\
- Walnut & $6 \%$ \\
- Corn & $6 \%$ \\
- Lemon & $3 \%$ \\
Carrot & $3 \%$ \\
\hline INORGANIC & \\
\hline Ring & $3 \%$ \\
Metal cross & $3 \%$ \\
\hline IATROGENIC & $6 \%$ \\
\hline Dental prosthesis & $3 \%$ \\
\hline Dental implant & \\
\hline Data are presented as number of patients (\%)
\end{tabular}

Pulmonary infection occurred in $51 \%$ of cases. Table 5 shows the microorganisms isolated in the FB samples. The most common microorganism was Actinomyces spp. which caused $9 \%$ of infections, followed by S. constellatus, S. aureus, M. morgagni and E. faecium, each accounting for $6 \%$. We did not observe unusual patterns of resistance: the bacteria were susceptible to the antibiotics most commonly prescribed (beta-lactams, cephalosporins and quinolones). The microbiological results of the bronchoscopy changed the antibiotic indication in only $16 \%$ of cases.

Table 5. Microbiological isolation in FB samples in patients with AFB.

\begin{tabular}{lc}
\hline \multicolumn{1}{c}{ Microorganism } & Occurrence \\
\hline No microorganism isolated & $49 \%$ \\
Actinomyces spp. & $9 \%$ \\
S. constellatus & $6 \%$ \\
S. aureus & $6 \%$ \\
M. morgagni & $6 \%$ \\
E. faecium & $6 \%$ \\
Serratia spp. & $3 \%$ \\
Acinetobacter baumannii & $3 \%$ \\
M. pneumoniae & $3 \%$ \\
Prevotella buccae & $3 \%$ \\
M. morgagni & $3 \%$ \\
Candida spp. & $3 \%$ \\
\hline
\end{tabular}

Data are presented as number of patients (\%).

\section{Discussion}

The prevalence of AFBs in this study was $0.25 \%$ of the bronchoscopies performed in adults. This figure is very similar to those available in the literature. A relatively recent bibliographical review reported a pooled prevalence of $0.24 \%$ (95\% CI 0.18-0.31), although the proportions in the different studies ranged from $0.16 \%$ to $0.33 \%$ [1]. These data show that although foreign body aspiration is uncommon in the adult population, it is a potential finding of an endoscopic airway examination. In a 
medium-sized unit, which performs around 500 bronchoscopies every year, there will be 1 or 2 yearly cases of AFB. Bronchoscopists therefore need to know how to manage this situation.

Different methods are available for removing AFBs. The preferred technique is normally RB, owing to factors such as better airway control, better protection of the mucous membranes, better management in central locations and larger instruments [15]. In recent years, however, more and more case series and systematic reviews have supported FB as the technique of choice, as it is simpler and generally more accessible, affords a much more complete examination of the airway and can be used even in patients with cervical injuries or who are intubated [1-4,11,16-19]. There are some very complex cases that may require RB owing to factors such as central location, large size or sharp parts. But in the absence of such factors, FB could be the method of choice for AFB extraction in adults.

The success rate of FB in our study was $94 \%$ which is relatively high compared to the rates presented in the literature. The reported efficacy of FB in AFB removal varies from $61 \%$ to $100 \%$, with a pooled rate of $89.6 \%$ (95\% CI 86.1-93.2) [1]. The relatively high success rate in our study is all the more relevant when we consider the size of our bronchoscopy unit. It may seem logical that only bronchoscopists in large units can obtain good results in such an infrequent procedure, as they are bound to treat more cases each year; yet in our study, only $6 \%$ of cases required subsequent RB to remove the foreign body. This proves that even small- or medium-sized units can achieve good results with FB.

Only $28 \%$ of our cases had an indication of suspected AFB. This is almost certainly because our hospital has no bronchoscopists working out of hours, meaning life-threatening cases, which cannot wait to be attended in the usual morning timeslot, are sent urgently to a nearby tertiary hospital for emergency bronchoscopy. In all likelihood, had we analysed these emergency bronchoscopies, the percentage of cases with suspected AFB would be slightly higher.

The safety data were excellent in this study, as no patients suffered FB-related complications. The rate of complications in previous studies is approximately $1 \%$, with bleeding being the most common complication. As well as being effective, then, FB is safe for AFB removal even in medium-sized units, a fact that further justifies the elective use of this technique [1,17].

In our study, most AFBs were removed with instruments specifically designed for foreign bodies: mainly gripping forceps but also baskets. However, up to $31 \%$ of cases were successfully resolved with conventional biopsy forceps which shows that the standard equipment of a respiratory endoscopy unit often suffices for this procedure. The devices for AFB removal described in the literature include standard biopsy forceps, forceps specifically designed for foreign bodies (alligator forceps), magnetic probes (for metal objects), several types of metal hooks, baskets (made of metal or synthetic materials), balloon catheters and even cryoprobes [16-18].

With FB there is always a risk that the AFB will migrate during removal. Factors such as size, irregularity, hardness and consistency of the foreign body can influence this risk. To prevent migration, bronchoscopists must try to fully surround the AFB if using a basket or apply firm pressure to effectively grip the AFB if using forceps. The main obstacle to take into account when extracting foreign bodies by FB is the glottis, which is the narrowest part of the airway. When the bronchoscope is inserted nasally, the nasal fossae are another critical point. Of course, sharp objects are more likely to damage the airway, meaning the consequences of migration are more serious. Perhaps RB should be the method of choice in these cases [16-18].

In our series there were very few cases of sharp AFBs and AFB migration. This may be because, as explained above, our hospital has no bronchoscopists or pulmonologists working out of hours, meaning life-threatening cases are sent urgently to a nearby tertiary hospital. The fact that we could not include emergency bronchoscopies referred to another hospital may constitute selection bias.

Regarding the access route used in $\mathrm{FB}$, bronchoscopists opted for the nasal route in a rather large proportion of cases (39\%) for several different reasons. Firstly, FB was indicated for suspected AFBs in only $28 \%$ of cases. For bronchoscopic airway examination, we normally chose the nasal entry route because it is associated with various advantages such as lesser lateral mobility of the bronchoscope 
and better patient tolerance. In most cases, AFBs were found incidentally during the examination. Airway foreign bodies that appeared to be regularly shaped and smaller in diameter than the far end of the bronchoscope were removed through the same nasal route. For AFBs judged to be irregularly shaped or larger than the bronchoscope, in the same procedure the examiner switched to the oral route for removal (58\% of cases), as the AFB may not have passed easily through the nasal fossa. The fact that we encountered no failures due to the difficulty removing AFBs through the nasal fossa would appear to support this approach.

With regard to patients' clinical characteristics, the most common presentation in our study was the triad of cough, sputum and fever which probably explains why the most common indication for bronchoscopy was pneumonia and why the duration of symptoms before presentation was relatively long. There is a danger, therefore, that symptoms can go unnoticed and be wrongly diagnosed as pneumonia, especially in older patients [20].

Most of our patients had radiological findings of alveolar infiltrate or atelectasis. While both findings are direct consequences of AFBs, there was a radiological suspicion of AFBs in only $18 \%$ of cases. In previous studies, this figure ranged from $25 \%$ to $47 \%$ of cases [1,4]. The nature and size of the foreign objects identified in each series could explain these differences: large and calcific density foreign bodies are easier to detect in a radiological examination than small or vegetable foreign bodies.

In this study, as in previous studies, most foreign objects were located in the right bronchial tree. Anatomical factors explain this universal tendency: the right main bronchus is wider and more vertical than the left main bronchus, and the carina lies left of the trachea midline [21].

Most AFBs found in our study were organic (more animal than vegetable); inorganic and iatrogenic foreign bodies were relatively infrequent. Although the numbers vary in the literature, inorganic and iatrogenic AFB are usually more common than organic AFB [1,3-6,10]. Two factors could explain our unusual results: firstly, our unit is not a specialised interventional pulmonology unit; and secondly, we do not have an on-call bronchoscopist. Specialised unit case series normally include more cases of inorganic and iatrogenic AFBs because these cases are referred to tertiary referral hospitals. Social and dietary factors associated with the place where the hospital is located may also influence the frequency of different AFB types. Finally, we must keep in mind the inherent variability of case series which usually include dozens of cases [1].

In our study, bronchial infection was reported in $51 \%$ of cases. The few studies that include this parameter report similar figures [4]. We considered the pulmonary infection to be relevant in all cases, because significant quantities of microorganisms were isolated with a quantitative culture technique and these findings were consistent with imaging test results and clinical features. Although bronchial infection is rarely considered in the literature, we consider it to be a very significant factor. Our findings in this regard show that foreign-body bronchial obstruction frequently causes infection, probably because it can inhibit natural defence mechanisms of the mucosa and airway such as coughing and ciliary beating. The types of microorganisms identified also suggest that they were probably related to the aspirated content. All patients with a pneumonia diagnosis had taken antibiotics before the bronchoscopy, which could have influenced the rate of positive cultures.

The microbiological results changed the antibiotic indication in only $16 \%$ of cases, since in the rest, antibiograms showed that the bacteria were susceptible to the empirically prescribed antibiotic. All this suggests that beta-lactam antibiotics constitute the best empirical treatment for these cases. Including bronchial infection highlights the utility of FB for microbiological diagnoses and can provide useful data on antibiotic resistance in each case, potentially improving patient prognosis.

\section{Conclusions}

The prevalence of AFBs was $0.25 \%$ of the bronchoscopies performed in adults.

We found FB to be a safe and effective technique for removing foreign bodies from the airways, with a success rate of $94 \%$ and no associated complications. 
The most common clinical presentation was the triad of cough, sputum and fever. The most frequent radiological findings were alveolar infiltrates and atelectasis.

Most AFBs were organic; inorganic and iatrogenic AFBs were relatively infrequent.

Bronchial infection occurred in around half of cases.

Author Contributions: Conceptualization, J.N.S.-C. and E.C.; methodology, E.C.; software, J.N.S.-C.; validation, J.N.S.-C. and E.C.; formal analysis, J.N.S.-C. and E.C.; investigation, V.M., S.V., A.M.P. and L.M.; data curation, A.M.P. and L.M.; writing-original draft preparation, V.M. and S.V.; writing-review and editing, J.N.S.-C.; visualization and supervision, J.N.S.-C. and E.C.; All authors have read and agreed to the published version of the manuscript.

Acknowledgments: The authors thank all the present and past workers of the Respiratory Medicine Section of Sant Joan d'Alacant University Hospital.

Conflicts of Interest: The authors declare no conflict of interest.

\section{References}

1. Sehgal, I.S.; Dhooria, S.; Ram, B.; Singh, N.; Aggarwal, A.N.; Gupta, D.; Behera, D.; Agarwal, R. Foreign Body Inhalation in the Adult Population: Experience of 25,998 Bronchoscopies and Systematic Review of the Literature. Respir. Care 2015, 60, 1438-1448. [CrossRef]

2. Hsu, A.A. Endoscopic intervention of lower airway foreign matter in adults-a different perspective. J. Thorac. Dis. 2015, 7, 1870-1877. [CrossRef]

3. Mise, K.; Jurcev Savicevic, A.; Pavlov, N.; Jankovic, S. Removal of tracheobronchial foreign bodies in adults using flexible bronchoscopy: Experience 1995-2006. Surg. Endosc. 2009, 23, 1360-1364. [CrossRef]

4. Ramos, M.B.; Fernández-Villar, A.; Rivo, J.E.; Leiro, V.; García-Fontán, E.; Botana, M.I.; Torres, M.L.; Cañizares, M.A. Extraction of airway foreign bodies in adults: Experience from 1987-2008. Interact. Cardiovasc. Thorac. Surg. 2009, 9, 402-405. [CrossRef] [PubMed]

5. Debeljak, A.; Sorli, J.; Music, E.; Kecelj, P. Bronchoscopic removal of foreign bodies in adults: Experience with 62 patients from 1974-1998. Eur. Respir. J. 1999, 14, 792-795. [CrossRef] [PubMed]

6. Sauret Valet, J. Cuerpos extraños. Arch. Bronconeumol. 2002, 38, 285-287. [CrossRef]

7. Killian, G. Meeting of the Society of Physicians of Freiburg. Munchen. Med. Wschr. 1898, 45, 378.

8. Panchabhai, T.S.; Mehta, A.C. Historical perspectives of bronchoscopy. Connecting the dots. Ann. Am. Thorac. Soc. 2015, 12, 631-641. [CrossRef] [PubMed]

9. Zavala, D.C.; Rhodes, M.L. Experimental removal of foreign bodies by fiberoptic bronchoscopy. Am. Rev. Respir. Dis. 1974, 110, 357-360. [CrossRef]

10. Donado Uña, J.R.; de Miguel Poch, E.; Casado López, M.E.; Alfaro Abreu, J.J. La fíbrobroncoscopia en la extracción de cuerpos extraños traqueobronquiales en adultos. Arch. Bronconeumol. 1998, 34, 76-81. [CrossRef]

11. Rodrigues, A.J.; Oliveira, E.Q.; Scordamaglio, P.R.; Gregório, M.G.; Jacomelli, M.; Figueiredo, V.R. Flexible bronchoscopy as the first-choice method of removing foreign bodies from the airways of adults. J. Bras. Pneumol. 2012, 38, 315-320. [CrossRef] [PubMed]

12. Gómez López, A.; García Luján, R.; de Miguel Poch, E. Broncoaspiración de cuerpos extraños. Caso clínico y revisión. Arch. Bronconeumol. 2015, 51, 358-359. [CrossRef] [PubMed]

13. Páez Codeso, F.M.; Dorado Galindo, A.; González Angulo, G.E. Broncoaspiración de cuerpo extraño odontológico. A propósito de tres casos. Arch. Bronconeumol. 2016, 52, 443-444. [CrossRef] [PubMed]

14. Du Rand, I.A.; Blaikley, J.; Booton, R.; Chaudhuri, N.; Gupta, V.; Khalid, S.; Mandal, S.; Martin, J.; Mills, J.; Navani, N.; et al. Summary of the British Thoracic Society guideline for diagnostic flexible bronchoscopy in adults. Thorax 2013, 68, 786-787. [CrossRef]

15. Limper, A.H.; Prakash, U.B. Tracheobronchial foreign bodies in adults. Ann. Intern. Med. 1990, 112, $604-609$. [CrossRef]

16. Hewlett, J.C.; Rickman, O.B.; Lentz, R.J.; Prakash, U.B.; Maldonado, F. Foreign body aspiration in adult airways: Therapeutic approach. J. Thorac. Dis. 2017, 9, 3398-3409. [CrossRef]

17. Blanco Ramos, M.; Botana-Rial, M.; García-Fontán, E.; Fernández-Villar, A.; Gallas Torreira, M. Update in the extraction of airway foreign bodies in adults. J. Thorac. Dis. 2016, 8, 3452-3456. [CrossRef] 
18. Fang, Y.F.; Hsieh, M.H.; Chung, F.T.; Huang, Y.K.; Chen, G.Y.; Lin, S.M.; Lin, H.C.; Wang, C.H.; Kuo, H.P. Flexible bronchoscopy with multiple modalities for foreign body removal in adults. PLoS ONE 2015, 10, e0118993. [CrossRef]

19. Cravo, J.; Marques, M.A.T. Endobronchial foreign bodies in adults_Flexible bronchoscopy as a first approach. Pulmonology 2018, 24, 198-199. [CrossRef]

20. Lin, L.; Lv, L.; Wang, Y.; Zha, X.; Tang, F.; Liu, X. The clinical features of foreign body aspiration into the lower airway in geriatric patients. Clin. Interv. Aging 2014, 9, 1613-1618. [CrossRef]

21. Minnich, D.J.; Mathisen, D.J. Anatomy of the Trachea, Carina, and Bronchi. Thorac. Surg. Clin. 2007, 17, 571-585. [CrossRef] [PubMed]

(C) 2020 by the authors. Licensee MDPI, Basel, Switzerland. This article is an open access article distributed under the terms and conditions of the Creative Commons Attribution (CC BY) license (http://creativecommons.org/licenses/by/4.0/). 